PROCEEDINGS OF THE

AMERICAN MATHEMATICAL SOCIETY

Volume 127, Number 8, Pages 2233-2243

S 0002-9939(99)04874-1

Article electronically published on April 9, 1999

\title{
REPRESENTATIONS OF INFINITE PERMUTATIONS BY WORDS (II)
}

\author{
RANDALL DOUGHERTY AND JAN MYCIELSKI \\ (Communicated by Ronald M. Solomon) \\ Dedicated to the memory of Roger C. Lyndon
}

\begin{abstract}
We present an argument (due originally to R. C. Lyndon) which completes the proof of the following theorem: Every free group word which is not a proper power can represent any permutation of an infinite set.
\end{abstract}

\section{INTRODUCTION}

Let $F$ be a free group, $w$ a member of $F, G$ any group, and $g$ a member of $G$. We say that $w$ can represent $g$ in $G$ iff there exists a homomorphism $h: F \rightarrow G$ such that $h(w)=g$. In other words, treating the generators of $F$ as unknowns, one can solve the equation $w=g$ in $G$. For any set $A$, we denote by $S(A)$ the group of all permutations of $A$.

The purpose of this paper is to give an alternative version, with some additional consequences, of part of the proof of the following theorem of Lyndon and Mycielski.

Theorem. If $w$ is not a proper power (i.e., $w \neq v^{n}$ for $v \in F, n>1$ ) and $A$ is infinite, then $w$ can represent every permutation $\pi \in S(A)$ in $S(A)$.

Several cases of this theorem were proved earlier. D. Silberger [12] showed it for $w=x^{p} y^{q}, p, q \neq 0$, and asked whether it is true in general. M. Droste [3] showed it for $w=w_{1} w_{2}$, where $w_{1}$ and $w_{2}$ have no variables in common, and for $w=x^{p} y^{-r} x^{q} y^{r}$ (and one can even require that the permutations used for $x$ and $y$ lie in certain specific conjugacy classes).

The cases of the theorem when the permutation $\pi$ to be represented has infinitely many cycles of the same size (and possibly any other cycles) were proved in Mycielski [10]. In particular, if $A$ is uncountable, then $\pi$ must have $|A|$ cycles of the same size and the theorem holds. R. C. Lyndon [7] proved the rest of the theorem by handling the following cases:

(*) $A$ is countable and either $\pi$ has arbitrarily large finite cycles or $\pi$ has an infinite cycle.

The authors have simplified, somewhat, Lyndon's proof (using the same idea), and we present it here. Unlike the proof of the cases treated in Mycielski [10],

Received by the editors November 1, 1997.

1991 Mathematics Subject Classification. Primary 20F05.

The first author was supported by NSF grant number DMS-9158092 and by a fellowship from the Sloan Foundation. 
which uses some deep results about the topology of Cayley complexes, the proof assuming $(*)$ is elementary; the present paper is self-contained. In the third section of this paper, we give modifications of the construction which produce representing permutations with additional properties; we discuss the problem of representing two permutations simultaneously by two given words; and we solve some problems stated in Mycielski [10].

Let us add that groups other than $S(A)$ have also been considered. Ore [11] showed that, if $n \geq 5$, then every element of the alternating group $A_{n}$ is a commutator, and he proved the same for $S(A)$ for infinite $A$. Droste [4] showed that, for $p, q \neq 0$, if $m$ is the largest squarefree divisor of $p q$ and $n \geq 4 m+1$, then $x^{p} y^{q}$ can represent every element of $A_{n}$. Brenner, Evans, and Silberger [1] improved this to $n \geq \max \left(5, \frac{5}{2} \log m\right)$. For related work on permutations and on other groups, such as $S L(n, \mathbf{R})$, see Ehrenfeucht et al. [5], Mycielski [8], and Mycielski [9].

\section{Proof of the theorem under assumption $(*)$}

We will write actions of permutations on the right: applying permutation $\pi$ to element $a$ gives element $a \pi$. So a composition $\pi_{1} \pi_{2}$ indicates first applying $\pi_{1}$, then $\pi_{2}$.

Let $F$ be a free group on the generators $x_{1}, \ldots, x_{n}$. (Examples will be given in terms of two generators, $x$ and $y$. Actually, the general case can be reduced to the case of two generators [10], but this would not simplify the proof here.) Let $w=w\left(x_{1}, \ldots, x_{n}\right)$ be a word in the generators $x_{1}, \ldots, x_{n}$ which is not a proper power in $F$, and let $\pi$ be a permutation of $A$ satisfying (*). We want to show that $w$ represents $\pi$ in $S(A)$; that is, there exist $\sigma_{1}, \ldots, \sigma_{n} \in S(A)$ such that $w\left(\sigma_{1}, \ldots, \sigma_{n}\right)=\pi$.

We will use the term letter, and the variable $r$, to denote a generator $x_{k}$ or an inverse generator $x_{k}^{-1}$. So $w$ is a product of letters, say $w=r_{1} r_{2} \cdots r_{l}$.

Clearly, we may assume that $w$ is a reduced word. In fact, since words which can be mapped to each other by automorphisms of $F$ (in particular, conjugate words) represent the same permutations, we may assume that $w$ is cyclically reduced. (See Proposition 3 of Silberger [12].) Also, the result is trivial if $w$ has length 1, so assume that $w$ has length greater than 1 . (Since $w$ is not a power, it follows that $w$ must use more than one of the generators.)

We will construct the desired solution in the form of a labeled directed graph $D_{\infty}$, with vertex set $V_{\infty}$. Each vertex will be labeled with one of the elements of $A$, and each such element will appear exactly once as a label, so the labeling will give a bijection between $A$ and $V_{\infty}$. Each edge will be labeled by one of the generators $x_{k}$. (There may be multiple edges with different labels joining the same pair of vertices.) For each $k$, every vertex will have exactly one $x_{k}$-edge leading from it and exactly one $x_{k}$-edge leading to it. This means that, for each $k$, the $x_{k}$-edges will induce a bijection from $V_{\infty}$ to $V_{\infty}$. Hence, we will be able to define $\sigma_{k} \in S(A)$ by the condition that the $x_{k}$-edge starting at the vertex labeled $a$ ends at the vertex labeled $a \sigma_{k}$. The goal is to construct $D_{\infty}$ so that the resulting permutations $\sigma_{1}, \ldots, \sigma_{n}$ satisfy $w\left(\sigma_{1}, \ldots, \sigma_{n}\right)=\pi$.

We will construct $D_{\infty}$ by starting with a larger labeled digraph which includes unlabeled vertices and does not have enough edges to define the permutations fully. Then, repeatedly identifying vertices in this digraph (gluing them together), we end up with the desired configuration $D_{\infty}$ in the limit. 


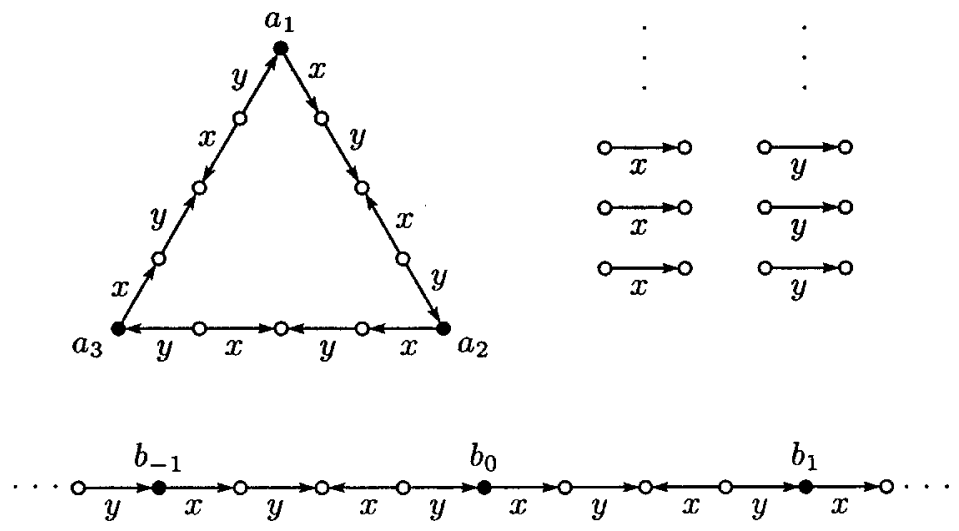

Figure 1. The initial labeled digraph for the construction.

The initial labeled digraph $D_{0}$, with vertex set $V_{0}$, is defined as follows. First, define " $x_{k}^{-1}$-edge from $v$ to $v^{\prime}$ " to mean an $x_{k}$-edge from $v^{\prime}$ to $v$; this makes it convenient to talk about $r$-edges without specifying whether $r$ is a generator or an inverse generator. Now, for each $a \in A, V_{0}$ contains distinct vertices $v_{a, i}$ for $0 \leq i<l$ (where $w=r_{1} r_{2} \cdots r_{l}$ ), and vertex $v_{a, 0}$ has label $a$ in $D_{0}$; the other vertices $v_{a, i}$ are unlabeled. For each $i$ such that $1 \leq i<l$, there is an $r_{i}$-edge from $v_{a, i-1}$ to $v_{a, i}$; also, there is an $r_{l}$-edge from $v_{a, l-1}$ to $v_{a \pi, 0}$. Finally, for each $k \leq n$, put into $D_{0}$ countably infinitely many pairs of (unlabeled) vertices $u, u^{\prime}$ with no edges except for an $x_{k}$-edge from $u$ to $u^{\prime}$. (We will call these free $x_{k}$-edges.) This completes the definition of $D_{0}$.

For example, Figure 1 shows what $D_{0}$ looks like for the case of two generators $x$ and $y$, where $w=x y x^{-1} y$ and $\pi$ consists of a 3-cycle $\left(a_{1} a_{2} a_{3}\right)$ and an infinite cycle $\left(\ldots b_{-1} b_{0} b_{1} b_{2} b_{3} \ldots\right)$.

The initial digraph $D_{0}$ has the following four properties, which will be maintained throughout the construction:

(1) Each $a \in A$ is a label for exactly one vertex.

(2) No vertex has more than one label.

(3) Each edge is labeled by exactly one of the generators $x_{k}$.

(4) Each vertex has at most one $x_{k}$-edge leading from it and at most one $x_{k}$-edge leading to it.

Property (4) holds in $D_{0}$ because $w$ is reduced and cyclically reduced.

In $D_{0}$ or any other digraph satisfying (4), the following definition makes sense. For a vertex $v$ and a letter $r$, let $v r$ be the end point of the $r$-edge that starts at $v$, if there is one; otherwise, let $v r$ be undefined. Then one can define $v r r^{\prime}=(v r) r^{\prime}$, and so on for longer words, so long as the requisite edges exist.

Another property that holds in $D_{0}$ and will hold in the later digraphs as well is:

(5) If vertex $v$ has label $a$, then $v w$ exists and has label $a \pi$.

It follows that $v w^{-1}$ also exists and has label $a \pi^{-1}$, because, if $v^{\prime}$ is the vertex with label $a \pi^{-1}$, then $v^{\prime} w$ must be the vertex with label $a$, namely $v$. It also follows that, if $v$ is unlabeled, then $v w$ and $v w^{-1}$ are unlabeled if they exist.

One can view (5) as saying that $D_{0}$ gives a solution to our problem, except that the resulting 'permutations' are one-to-one maps not on $A$ but on subsets of 
some set including $A$. The remaining part of the construction must get rid of the extraneous elements.

We will do this by a sequence of identifications. Suppose $D$ is a digraph with vertex set $V$ and labels of the sort that $D_{0}$ has. Then, given a function $g: V \rightarrow V^{\prime}$, we can produce a labeled digraph $D^{\prime}$ on vertex set $V^{\prime}$ : if $v \in V$ has label $a$ in $D$, then $g(v)$ gets label $a$ in $D^{\prime}$; if there is an $x_{k}$-edge from $u$ to $v$ in $D$, then there is an $x_{k}$-edge from $g(u)$ to $g(v)$ in $D^{\prime}$. (If there is also an $x_{k}$-edge from $u^{\prime}$ to $v^{\prime}$ where $g\left(u^{\prime}\right)=g(u)$ and $g\left(v^{\prime}\right)=g(v)$, then put only one $x_{k}$-edge from $g(u)$ to $g(v)$ in $D^{\prime}$. However, if the edge from $u^{\prime}$ to $v^{\prime}$ has a different label, $x_{k^{\prime}}$, then there will be multiple edges from $g(u)$ to $g(v)$ in $D^{\prime}$ with different labels.) We will call $D^{\prime}$ the $g$-image of $D$. If $g(v)=g\left(v^{\prime}\right)$, then $v$ and $v^{\prime}$ have been identified in $D^{\prime}$.

Properties (1) and (3) are preserved under all identifications ( $g$-images), but one has to make extra assumptions about $g$ in order for properties (2) and (4) to be preserved. Note that, if (4) holds in $D$ and $D^{\prime}$, then, for any $v \in V$ and any word $\bar{w}$, if $v \bar{w}$ exists in $D$, then $g(v) \bar{w}$ exists in $D^{\prime}$ and is equal to $g(v \bar{w})$. Hence, in this case, property (5) is also preserved under the identification.

If we also have $g^{\prime}: V^{\prime} \rightarrow V^{\prime \prime}$, and $D^{\prime \prime}$ is the $g^{\prime}$-image of $D^{\prime}$, then $D^{\prime \prime}$ is the $\left(g^{\prime} \circ g\right)$-image of $D$.

Starting with $D_{0}$, we will produce a sequence of digraphs $D_{0}, D_{1}, D_{2}, \ldots$; for each $s>0, D_{s}$ will be the $g_{s}$-image of $D_{s-1}$ for some surjection $g_{s}: V_{s-1} \rightarrow V_{s}$. Let $f_{s}: V_{0} \rightarrow V_{s}$ be the composition $g_{s} \circ \cdots \circ g_{1}$ (and let $f_{0}$ be the identity map on $V_{0}$ ); then $D_{s}$ will be the $f_{s}$-image of $D_{0}$.

Each identifying map $g_{s}$ will be produced in the following way. Suppose we have vertices $u_{1}, \ldots, u_{m}$ and $v_{1}, \ldots, v_{m}$ in $D_{s-1}$ such that:

- The vertices $u_{1}, \ldots, u_{m}, v_{1}, \ldots, v_{m}$ are all distinct.

- There is no $j$ such that $u_{j}$ and $v_{j}$ are both labeled.

- For any $j$ and any letter $r$, if there are $r$-edges starting at both $u_{j}$ and $v_{j}$, then there is a $j^{\prime}$ such that these $r$-edges end at $u_{j^{\prime}}$ and $v_{j^{\prime}}$, respectively.

Then we can let $V_{s}=V_{s-1} \backslash\left\{u_{1}, \ldots, u_{m}\right\}$, and define $g_{s}: V_{s-1} \rightarrow V_{s}$ by $g_{s}\left(u_{j}\right)=v_{j}$ and $g_{s}(v)=v$ for all other $v$. We call this a basic identification, and say that $D_{s}$ is obtained from $D_{s-1}$ by identifying $u_{1}, \ldots, u_{m}$ with $v_{1}, \ldots, v_{m}$. The assumptions above imply that properties (2) and (4) (and hence all of (1)-(5)) are preserved by basic identifications.

Since we will be producing the digraphs $D_{s}$ by a sequence of basic identifications, the following property will be preserved automatically:

(6) Each vertex in $D_{s}$ has only finitely many $f_{s}$-preimages in $D_{0}$, and only finitely many vertices in $D_{s}$ have more than one $f_{s}$-preimage.

Equivalently, only finitely many original vertices $v_{0} \in V_{0}$ have been identified with another vertex by the map $f_{s}$. In particular, for each $k$, there will be infinitely many free $x_{k}$-edges in $D_{0}$ for which neither vertex is identified with anything else by $f_{s}$; these edges become free $x_{k}$-edges in $D_{s}$.

Let $v_{0}$ be a vertex in a labeled digraph satisfying (1)-(4). Then one can try to construct a path by starting at $v_{0}$, taking the $r_{1}$-edge to $v_{1}=v_{0} r_{1}$, then taking the $r_{2}$-edge to $v_{2}=v_{1} r_{2}$, and so on. If one reaches $v_{l}=v_{l-1} r_{l}=v_{0} w$, then one can cycle again through $w$ to define $v_{l+1}=v_{l} r_{1}, v_{l+2}=v_{l} r_{1} r_{2}$, and so on. One may be able to continue this forever, or it may terminate when one reaches a vertex $v_{M}$ at which no $r_{(M \bmod l)+1}$-edge starts. In either case, we call the resulting path $v_{0}, v_{1}, \ldots$ the forward $w$-path rooted at $v_{0}$. 
Similarly, one can define the backward $w$-path rooted at $v_{0}$ to be the forward $w^{-1}$ path rooted at $v_{0}$, which will be of the form $v_{0}, v_{-1}, v_{-2}, \ldots$ where $v_{-1}=v_{0} r_{l}^{-1}$, $v_{-2}=v_{0} r_{l}^{-1} r_{l-1}^{-1}, v_{-l}=v_{0} w^{-1}$, and so on, continuing forever or until the required $r_{i}^{-1}$-edge fails to exist. The combined path $\ldots, v_{-2}, v_{-1}, v_{0}, v_{1}, v_{2}, \ldots$ is called the $w$-path rooted at $v_{0}$.

Note that a $w$-path does not double back on itself (use an edge in the forward direction and then immediately use this edge backward, or vice versa), because $w$ is reduced and cyclically reduced.

In $D_{0}$, if vertex $v$ has label $a$, then $v w^{j}$ is defined for all integers $j$ (and has label $a \pi^{j}$ ), so the $w$-path rooted at $v$ is infinite in both directions. (Note that this infinite path may be periodic, a finite cycle traversed infinitely many times.) The final property we will need for the digraphs $D_{s}$ is that these are the only infinite w-paths.

(7) For any unlabeled vertex $v$, the $w$-path rooted at $v$ is finite (in both directions).

In other words, for any unlabeled $v, v w^{j}$ exists for only finitely many integers $j$.

The basic identifications used to produce the digraphs $D_{s}$ will be carefully chosen so as to preserve property (7) as well as (1)-(6). First, though, we have to see that this property holds in $D_{0}$. Clearly an infinite $w$-path cannot occur within the free $x_{k}$-edges (since $w$-paths cannot double back). The other components of $D_{0}$ are themselves $w$-paths (cyclic or doubly infinite) rooted at labeled vertices. We must see that, if we try to follow one of these paths 'out of phase' (i.e., starting at an unlabeled vertex) as a $w$-path, then we will only succeed for finitely many steps.

In fact, we will show that the forward $w$-path rooted at an unlabeled vertex $u$ in one of these components of $D_{0}$ must have fewer than $l$ edges. Recalling the notation for the elements of $V_{0}$, we must have $u=v_{a, i}$ for some $a \in A$ and some $i$, $0<i<l$. If the forward $w$-path rooted at $u$ lasts as long as $l$-edges, reaching from $u_{0}=u$ to $u_{l}=u w$, then, since this path cannot double back on itself in $D_{0}$, it must follow the paths in $D_{0}$ either forward $\left(u_{1}=v_{a, i+1}, u_{2}=v_{a, i+2}\right.$, and so on) or backward $\left(u_{1}=v_{a, i-1}, u_{2}=v_{a, i-2}\right.$, and so on). In the former case, we must have $r_{1}=r_{i+1}, r_{2}=r_{i+2}, \ldots, r_{l-i}=r_{l}, r_{l-i+1}=r_{1}, \ldots, r_{l}=r_{i}$; this means that the word $w$ is periodic with period $\operatorname{gcd}(i, l)$, which contradicts the assumption that $w$ is not a proper power in $F$. In the latter case, we get $r_{1}=r_{i}^{-1}, r_{2}=r_{i-1}^{-1}$, and so on. If $i$ is odd, this gives $r_{(i+1) / 2}=r_{(i+1) / 2}^{-1}$, which is impossible; if $i$ is even, we get $r_{(i / 2)+1}=r_{i / 2}^{-1}$, which is impossible because $w$ is reduced. Therefore, $u_{l}$ cannot exist.

The same argument works for the backward $w$-path rooted at $u$, so this must have fewer than $l$ edges as well. Therefore, $D_{0}$ satisfies all of the properties (1)-(7).

The sequence of identifications will be set up so as to take care of the following requirements:

(a) Every vertex eventually has a full set of edges attached: for each $v_{0} \in V_{0}$ and each letter $r$, there is a stage $s$ such that $f_{s}\left(v_{0}\right) r$ exists in $D_{s}$.

(b) Every vertex is eventually identified with a labeled vertex: for each $v_{0} \in V_{0}$, there is a stage $s$ such that $f_{s}\left(v_{0}\right)$ has a label in $D_{s}$.

Each of (a) and (b) can be thought of as a countably infinite list of requirements; (b) gives a requirement for each vertex $v_{0}$, and (a) gives a requirement for each pair $\left(v_{0}, r\right)$. Since we have infinitely many stages $s=1,2,3, \ldots$ available, it is easy 
to arrange a schedule (map requirements to stages) so that just one requirement needs to be taken care of at each stage. Note that, once a requirement has been taken care of at stage $s\left(f_{s}\left(v_{0}\right)\right.$ has an $r$-edge or a label $)$, it will remain true at stages $s^{\prime}>s$.

Suppose that we have defined $D_{s-1}$ so that properties (1)-(7) hold and, at stage $s$, we must take care of the requirement that $f_{s}\left(v_{0}\right) r$ exists. Let $v=f_{s-1}\left(v_{0}\right)$. If $v r$ exists in $D_{s-1}$, we do not need to do anything, so just let $g_{s}$ be the identity map on $V_{s-1}$. If $v r$ does not exist, then find a free $r$-edge in $D_{s-1}$, say the edge from $u$ to $u^{\prime}$, where $u, u^{\prime} \neq v$ (this is possible because $D_{s-1}$ has more than one free $r$-edge). Obtain $D_{s}$ from $D_{s-1}$ by identifying $u$ with $v$. This is clearly a basic identification, and $f_{s}\left(v_{0}\right)=g_{s}(v)=g_{s}(u)$ is the starting point of an $r$-edge in $D_{s}$. Hence, $D_{s}$ and $f_{s}$ defined in this way satisfy (1)-(6). To see that (7) also holds in $D_{s}$, note that vertex $u^{\prime}$ is incident to only one edge in $D_{s}$, so it cannot lie on any $w$-path except possibly at an end of that path. Hence, if $\bar{v}$ is an unlabeled vertex such that the forward $w$-path rooted at $\bar{v}$ is infinite, then the forward $w$ path rooted at $\bar{v} w$ is also infinite and avoids $u^{\prime}$. But $D_{s}$ with $u^{\prime}$ omitted is actually included in $D_{s-1}$, so there is an infinite $w$-path in $D_{s-1}$ rooted at $\bar{v} w$, which is an unlabeled vertex. This contradicts $(7)$ for $D_{s-1}$. A similar contradiction arises if the backward $w$-path rooted at $\bar{v}$ is infinite, so (7) holds in $D_{s}$. Therefore, $D_{s}$ has all of the desired properties.

Now suppose that the requirement to be taken care of at stage $s$ is that $f_{s}\left(v_{0}\right)$ has a label. Again, if $f_{s-1}\left(v_{0}\right)$ already has a label in $D_{s-1}$, we can just let $g_{s}$ be the identity map, so assume that $f_{s-1}\left(v_{0}\right)$ has no label. Let $v=f_{s-1}\left(v_{0}\right)$. By (7), we can define $\bar{v}_{m}, \bar{v}_{m+1}, \ldots, \bar{v}_{M}$ to be the $w$-path rooted at $v$, where $m \leq 0 \leq M$ and $\bar{v}_{0}=v$.

Claim. There is a labeled vertex $u_{0} \in V_{0}$ such that, if $\ldots, u_{-2}, u_{-1}, u_{0}, u_{1}, u_{2}, \ldots$ is the $w$-path in $D_{0}$ rooted at $u_{0}$, then the vertices $u_{m-2 l}, u_{m-2 l+1}, \ldots, u_{M+2 l}$ are distinct, and none of them is identified with any other vertex or mapped to one of the vertices $\bar{v}_{j}$ by $f_{s-1}$.

Proof. Let $N$ be the number of vertices in $D_{0}$ which are identified with another vertex or mapped to some $\bar{v}_{j}$ by $f_{s-1}$; by $(6)$, this is finite. By $(*), \pi$ has a cycle which is so long that the corresponding $w$-path in $D_{0}$ includes $N+1$ disjoint subpaths of the form $u_{m-2 l}, u_{m-2 l+1}, \ldots, u_{M+2 l}$ with $u_{0}$ labeled; at least one of these subpaths contains none of the $N$ 'bad' vertices.

So find such a vertex $u_{0} \in V_{0}$, and let $\bar{u}_{j}=f_{s-1}\left(u_{j}\right)$ for $m-2 l \leq j \leq M+2 l$. Then $\bar{u}_{m-2 l}, \bar{u}_{m-2 l+1}, \ldots, \bar{u}_{M+2 l}$ is part of the $w$-path rooted at $\bar{u}_{0}$ in $D_{s-1}$, and the $w$-path edges are the only edges incident to these vertices. Obtain $D_{s}$ from $D_{s-1}$ by identifying $\bar{u}_{m}, \bar{u}_{m+1}, \ldots, \bar{u}_{M}$ with $\bar{v}_{m}, \bar{v}_{m+1}, \ldots, \bar{v}_{M}$. Since $\bar{v}_{m}, \bar{v}_{m+1}, \ldots, \bar{v}_{M}$ is the entire $w$-path rooted at the unlabeled vertex $v=\bar{v}_{0}$ (and hence all of the vertices $\bar{v}_{j l}=v w^{j}$ are unlabeled), this is a basic identification, so $D_{s}$ satisfies (1)-(6). Now we will show that (7) also holds.

Suppose to the contrary that property (7) fails in $D_{s}$; say the forward $w$-path rooted at the unlabeled vertex $\tilde{v}$ is infinite. Then the forward $w$-path rooted at $\tilde{v} w^{2}$ (which is all but the first $2 l$ edges of the preceding path) is also infinite. This latter path must contain at least one of the vertices $\bar{u}_{m-1}$ and $\bar{u}_{M+1}$, because $D_{s}$ without these two vertices is included in $D_{s-1}$, which satisfies $(7)$. So the forward $w$-path rooted at $\tilde{v}$ must include as a subpath one of the two finite paths $\bar{u}_{m-2 l}, \ldots, \bar{u}_{m-1}$ 
and $\bar{u}_{M+1}, \ldots, \bar{u}_{M+2 l}$, either forward or backward. Say, for instance, that it includes $\bar{u}_{M+1}, \ldots, \bar{u}_{M+2 l}$ traced forward. Then one of the vertices $\bar{u}_{M+i}$ for $1 \leq i \leq l$ must be $\tilde{v} w^{j}$ for some $j$, and we also have $\tilde{v} w^{j} r_{1}=\bar{u}_{M+i+1}, \tilde{v} w^{j} r_{1} r_{2}=\bar{u}_{M+i+2}$, and so on to $\tilde{v} w^{j+1}=\bar{u}_{M+i+l}$. But vertices $u_{M+i}, \ldots, u_{M+i+l}$ have not been identified with any other vertices by $f_{s}$, so we must have $u_{M+i} w=u_{M+i+l}$ in $D_{0}$, and $u_{M+i}$ is unlabeled (because $\tilde{v} w^{j}$ is unlabeled in $D_{s}$ ). This contradicts the earlier statement that a forward $w$-path in $D_{0}$ rooted at an unlabeled vertex must have length less than $l$. A similar contradiction arises in the other cases here, so the forward $w$-path rooted at $\tilde{v}$ must be finite. The same argument works for the backward $w$-path, so (7) holds for $D_{s}$.

This completes the definition of the digraphs $D_{s}$ and mappings $f_{s}$ and $g_{s}$. We must now see how to obtain a limit digraph $D_{\infty}$ in which all of the identifications have been made.

Since we have sets $V_{s}$ and mappings $g_{s}: V_{s-1} \rightarrow V_{s}$, we can define the direct limit in the usual way. (In fact, since the mappings $g_{s}$ are surjective, the definition is a bit simpler than usual.) So we get a set $V_{\infty}$, a surjection $f_{\infty}: V_{0} \rightarrow V_{\infty}$, and mappings $g_{s, \infty}: V_{s} \rightarrow V_{\infty}$ such that $f_{\infty}=g_{s, \infty} \circ f_{s}$. Let $D_{\infty}$ be the $f_{\infty}$-image of $D_{0}$; this is the same as the $g_{s, \infty}$-image of $D_{s}$, for any $s$.

It remains to show that $D_{\infty}$ has the properties initially specified for it. Since no two labeled vertices in $D_{0}$ were ever identified in $D_{s}$, each vertex in $D_{\infty}$ has at most one label. Since every vertex of $D_{0}$ was eventually identified with a labeled vertex, each vertex of $D_{\infty}$ has exactly one label. For each letter $r$, property (4) ensures that no vertex in $D_{s}$ has more than one $r$-edge leading from it, so the same holds for $D_{\infty}$; and requirements (a) ensure that each vertex of $D_{\infty}$ actually does have an $r$-edge leading from it. Therefore, the permutations $\sigma_{k} \in S(A)$ are well-defined. Finally, property (5) holds in $D_{\infty}$ because it holds in $D_{s}$, so the permuations $\sigma_{k}$ satisfy $w\left(\sigma_{1}, \ldots, \sigma_{n}\right)=\pi$. This completes the proof of the theorem.

In the cases treated in Mycielski [10] (where $(*)$ may fail), the representation of $\pi$ by $w$ is relatively simple. It consists of disjoint connected components, each of which is obtained by starting with the Cayley graph of a group given by a relation of the form $w^{n}=e$, and taking a certain natural cover followed by a homomorphism. In the present paper, the structure $D_{\infty}$ is much more irregular. Moreover, there is a great deal of arbitrariness in the structure, because of the choices made at each stage of the construction.

As we will see in the next section, though, these choices can provide useful flexibility, allowing us to produce permutations $\sigma_{k}$ satisfying $w\left(\sigma_{1}, \ldots, \sigma_{n}\right)=\pi$ with additional desirable properties. (Such flexibility is the reason that we introduced free $x_{k}$-edges, instead of attaching additional edges to the labeled vertices in the initial configuration $D_{0}$ so as to immediately take care of requirements (a) for these vertices.)

\section{Extensions, REMARKS, AND PROBlems}

1. Using single-cycle permutations. By adding extra requirements to the construction, one can ensure that the permutations in the representation of $\pi$ by $w$ have additional properties. For instance, one can show: If $(*)$ holds, and $w=$ $w\left(x_{1}, \ldots, x_{n}\right)$ is a word of length $l>1$ which is not a proper power in the free group $F$, then there exist $\sigma_{1}, \ldots, \sigma_{n} \in S(A)$ satisfying $w\left(\sigma_{1}, \ldots, \sigma_{n}\right)=\pi$ such that each $\sigma_{k}$ is a single infinite cycle moving all elements of $A$. Furthermore, we may 
ensure that, for any $a \in A$, the $2 n$ elements $a \sigma_{k}^{ \pm 1}$ for $k=1, \ldots, n$ are distinct, unless $l=2$ and $\pi$ has a fixed point.

This can be achieved as follows. We can define the $x_{k}$-path rooted at $v$ just as we did for $w$-paths (in fact, more easily). Now we impose two additional properties to be maintained during the construction:

(8) For all $k$ and all vertices $v$, the $x_{k}$-path rooted at $v$ is finite.

(9) For all vertices $v$ and all distinct letters $r_{1}$ and $r_{2}, v r_{1} \neq v r_{2}$.

Property (8) holds for $D_{0}$ because $w$ must use more than one of the generators; property (9) also holds for $D_{0}$ unless $l=2$ and $\pi$ has a fixed point (in which case we do not impose this property). It is not hard to see that the identifications we performed in order to meet requirements (a) and (b) preserve these two new properties.

We also add a third list of requirements to be met during the construction:

(c) All $x_{k}$-paths are eventually joined together: for all $k$ and all original vertices $v_{0}, v_{0}^{\prime} \in V_{0}$, there is a stage $s$ such that $f_{s}\left(v_{0}^{\prime}\right)$ lies on the $x_{k}$-path rooted at $f_{s}\left(v_{0}\right)$ in $D_{s}$.

There is still no problem arranging a schedule so that each stage takes care of one requirement and all requirements are eventually taken care of. Suppose that at stage $s$ we need to take care of requirement (c) for $k$ and $v_{0}, v_{0}^{\prime}$. If $f_{s-1}\left(v_{0}^{\prime}\right)$ is already on the $x_{k}$-path rooted at $f_{s-1}\left(v_{0}\right)$, do nothing at stage $s$ (let $g_{s}$ be the identity map). Otherwise, let $t$ be the last vertex on the $x_{k}$-path rooted at $f_{s-1}\left(v_{0}\right)$, and let $t^{\prime}$ be the first vertex on the $x_{k}$-path rooted at $f_{s-1}\left(v_{0}^{\prime}\right)$. Let $\left(u_{i}^{\prime}, u_{i}\right)$ for $i=1, \ldots, l$ be distinct free $x_{k}$-edges in $D_{s-1}$. Construct $D_{s}$ from $D_{s-1}$ by identifying $t, u_{1}, \ldots, u_{l}$ with $u_{1}^{\prime}, \ldots, u_{l}^{\prime}, t^{\prime}$. The effect of this is to link up the formerly separate $x_{k}$-paths into one path with $l$ new intermediate edges. This clearly will take care of the specified requirement (c), and it is not hard to show that properties (1)-(9) are preserved. (For (7), note that a $w$-path cannot contain $l$ consecutive $x_{k}$-edges or $x_{k}^{-1}$-edges.)

The new requirements (c) ensure that, in $D_{\infty}$, there is just a single $x_{k}$-path containing all of the vertices, so $\sigma_{k}$ is a cycle moving all elements of $A$. And property (9) ensures that $v r_{1} \neq v r_{2}$ for all $v \in V_{\infty}$ and all letters $r_{1} \neq r_{2}$, so the corresponding property holds for the permutations $\sigma_{k}$.

If $(*)$ fails, then it may not be possible to represent $\pi$ by $w$ using permutations which are infinite cycles moving all elements of the countable set $A$. In fact, this can fail even when $w$ is just $x y$; see below. But one can ask for weaker conditions, and we do not know whether these conditions can be met assuming that $A$ is countable but $(*)$ fails. For instance, can one always represent $\pi$ by $w$ using permutations such that the corresponding digraph is connected (weakly; that is, without regard to the orientation of the edges)?

2. Products of two cycles. A problem of Gale [6] asks whether every permutation of a countable set can be expressed as a product of two cycles. Gale notes that this is possible for a permutation of a finite set, and hence for a permutation of a countable set which only moves finitely many elements.

It turns out that one can modify the methods used here to solve Gale's problem. In fact, one can show: if $\pi$ is a permutation of the countable set $A$ which moves infinitely many elements of $A$, then $\pi$ can be expressed as a product of two infinite cycles, each of which moves all elements of $A$. Some changes are needed in the 
argument, because the hypothesis here is weaker than $(*)$; instead of starting with a larger digraph and identifying vertices, one starts with a smaller digraph and adds edges. The full construction is given in Dougherty [2]. F. Galvin independently found a similar proof of this result.

Note that a permutation of the countably infinite set $A$ which only moves finitely many elements of $A$ need not be expressible as a product of two cycles moving all elements of $A$; in fact, there can be no such expression if the finite permutation is odd. Galvin has shown that any even permutation of an $n$-element set can be expressed as a product of two $n$-cycles, and it follows that any even finite permutation of $A$ can be written as a product of two cycles moving all elements of $A$.

3. Representing two permutations simultaneously. Suppose that we have two words $w_{1}, w_{2}$ on the generators $x_{1}, \ldots, x_{n}$, and we want to solve two equations $w_{1}=\pi_{1}$ and $w_{2}=\pi_{2}$ simultaneously. What are the conditions on $w_{1}$ and $w_{2}$ such that this would be possible for all $\pi_{1}, \pi_{2} \in S(A)$ ?

First, suppose that $\pi_{1}$ and $\pi_{2}$ satisfy the analogue of $(*)$, which is:

(**) $A$ is countable and, for every finite set $W$ of reduced words in the letters $\pi_{j}$ and $\pi_{j}^{-1}$, there exists $a \in A$ such that the elements $a \omega$ for $\omega \in W$ are distinct.

One way of phrasing this is that $\pi_{1}$ and $\pi_{2}$ act locally freely on arbitrarily large neighborhoods in $A$.

The methods used here can be extended to show that the words $w_{1}$ and $w_{2}$ can simultaneously represent any pair of permutations $\left(\pi_{1}, \pi_{2}\right)$ satisfying $(* *)$, provided that $w_{1}$ and $w_{2}$ have the following properties:

(i) $w_{1} w_{2} \neq w_{2} w_{1}$ in $F$.

(ii) The subgroup $\left\langle w_{1}, w_{2}\right\rangle$ of $F$ is pure; that is, if $u \in\left\langle w_{1}, w_{2}\right\rangle$ and $u$ is a $k$-th power in $F$, then $u$ is a $k$-th power in $\left\langle w_{1}, w_{2}\right\rangle$.

(iii) If $u_{1}, u_{2} \in\left\langle w_{1}, w_{2}\right\rangle$ and $u_{1}, u_{2}$ are conjugate in $F$, then they are conjugate in $\left\langle w_{1}, w_{2}\right\rangle$.

Properties (ii) and (iii) are equivalent to: If $T$ is the subtree of the Cayley graph of $F$ (using the standard generators) spanned by $\left\langle w_{1}, w_{2}\right\rangle$, then any out-of-phase copy of $T$ (i.e., one not rooted at a member of $\left\langle w_{1}, w_{2}\right\rangle$ ) has only a finite overlap with $T$. They are also equivalent to the statement that the subgroup $H=\left\langle w_{1}, w_{2}\right\rangle$ of $F$ has trivial intersection with any conjugate $g H^{-1}$ for $g \in F \backslash H$; such a subgroup is called (in other contexts) a Frobenius complement.

Furthermore, one can show that properties (i)-(iii) are necessary in order for $\left(w_{1}, w_{2}\right)$ to represent any pair $\left(\pi_{1}, \pi_{2}\right)$ satisfying $(* *)$. This is clear for (i), since pairs $\left(\pi_{1}, \pi_{2}\right)$ need not commute. If (ii) fails, let $u=u\left(w_{1}, w_{2}\right)$ be a counterexample of minimal length. Then $u$ is not a proper power in $\left\langle w_{1}, w_{2}\right\rangle$, so one can let $\rho \in S(A)$ be a single infinite cycle, and apply the theorem to find $\pi_{1}, \pi_{2} \in S(A)$ such that $u\left(\pi_{1}, \pi_{2}\right)=\rho$. In fact, by adding extra requirements to the construction, one can ensure that $\pi_{1}$ and $\pi_{2}$ satisfy $(* *)$. But $\left(w_{1}, w_{2}\right)$ cannot represent $\left(\pi_{1}, \pi_{2}\right)$ if $u$ is a proper power in $F$, since $\rho$ is not a proper power in $S(A)$. Similarly, if (iii) fails for $u_{1}\left(w_{1}, w_{2}\right)$ and $u_{2}\left(w_{1}, w_{2}\right)$, then one can find $\pi_{1}$ and $\pi_{2}$ satisfying (**) such that $u_{1}\left(\pi_{1}, \pi_{2}\right)$ and $u_{2}\left(\pi_{1}, \pi_{2}\right)$ are not conjugate in $S(A)$. (We may assume that the cyclically reduced form of $u_{1}$ as a word in $w_{1}, w_{2}$ is no longer than that of $u_{2}$. Note that $u_{1}$ is not conjugate to $u_{2}^{-1}$ even in $F$, because $u_{1}$ is conjugate to $u_{2}$ in $F$ and no non-identity element of $F$ is conjugate to its own inverse. Therefore, $u_{1}$ is not conjugate to any power of $u_{2}$ in $\left\langle w_{1}, w_{2}\right\rangle$. Now let $A$ be the set of right cosets 
of $\left\langle u_{2}\right\rangle$ in $\left\langle w_{1}, w_{2}\right\rangle$, and define $\pi_{i} \in S(A)$ by $a \pi_{i}=a w_{i}$. Then $u_{2}\left(\pi_{1}, \pi_{2}\right)$ has a fixed point but $u_{1}\left(\pi_{1}, \pi_{2}\right)$ doesn't.) Hence, $\left(w_{1}, w_{2}\right)$ cannot represent $\left(\pi_{1}, \pi_{2}\right)$.

The same results hold for larger simultaneous systems, when one wants to use words $\left(w_{1}, \ldots, w_{m}\right)$ to represent permutations $\left(\pi_{1}, \ldots, \pi_{m}\right)$; this is possible for all $\pi_{1}, \ldots, \pi_{m}$ satisfying $(* *)$ if and only if $w_{1}, \ldots, w_{m}$ satisfy the versions of properties (i)-(iii) for $m$. In (ii) and (iii), just replace $\left\langle w_{1}, w_{2}\right\rangle$ with $\left\langle w_{1}, \ldots, w_{m}\right\rangle$; the new version of (i) is:

(i) $w_{1}, \ldots, w_{m}$ freely generate $\left\langle w_{1}, \ldots, w_{m}\right\rangle$ in $F$.

However, matters are more difficult if one wants to use $w_{1}$ and $w_{2}$ to represent all pairs $\left(\pi_{1}, \pi_{2}\right)$, not just those satisfying $(* *)$. Here are three examples where $w_{1}$ and $w_{2}$ satisfy (i)-(iii) but do not represent $\left(\pi_{1}, \pi_{2}\right)$.

$$
\begin{aligned}
& w_{1}=x y, \quad w_{2}=y^{2} x^{2} ; \quad \pi_{1}=\text { identity } \neq \pi_{2} . \\
& w_{1}=x, \quad w_{2}=y^{2} x y ; \quad \pi_{1}=\text { identity, } \pi_{2}=(a b c) \text {. } \\
& w_{1}=x, \quad w_{2}=y^{2} x y^{-1} ; \quad \pi_{1}=(a b), \quad \pi_{2}=(b c) .
\end{aligned}
$$

One can use these to obtain additional necessary conditions on $w_{1}$ and $w_{2}$ in order for them to represent all pairs $\left(\pi_{1}, \pi_{2}\right)$. The only sufficient condition the authors know of at present is $\left\langle w_{1}, w_{2}\right\rangle=\langle x, y\rangle$.

4. Intersections of Cayley cycles. We take this opportunity to note that the problem stated on page 239 of Mycielski [10] has a negative solution. Let $G$ be the Cayley graph of a group presented by one relation, and assume that the relator word is reduced and cyclically reduced. By a basic cycle, we mean any cycle in $G$ obtained by spelling the relator word of $G$, beginning at any vertex of $G$; if the starting vertex is $a$ and the relator word is $w=r_{1} r_{2} \ldots r_{l}$, then the basic cycle is the closed curve

$$
I L(a)=a, a r_{1}, a r_{1} r_{2}, \ldots, a r_{1} r_{2} \cdots r_{l-1}, a .
$$

(By a theorem of Weinbaum [13], the basic cycles of one-relator groups are always simple closed curves.) Then the intersection of two basic cycles of a onerelator group presentation may be disconnected. For example, the presentation $\langle a, b ; a b a b a=e\rangle$ has basic cycles $I L(e)=e, a, a b, a b a, a b a b, e$ and $I L(a)=a, a^{2}, a^{2} b$, $a^{2} b a, a^{2} b a b, a$. Since $a^{2} b a b=e$ and $a b a=a^{2} b$ (this follows from $\left.a b=a b a b a b a=b a\right)$, the intersection $I L(e) \cap I L(a)$ has two connected components, the edge $\{e, a\}$ and the singleton $\left\{a^{2} b\right\}$.

By a membrane we mean a closed two-dimensional cell whose boundary is a basic cycle. There are one-relator group presentations in which the union of some pair of membranes is an orientable (cylindrical) strip or a Möbius strip. Examples are $\langle a, b ; a b a a b a a=e\rangle$ and $\left\langle a, b, c ;\left(a b c b^{-1}\right)^{2} a=e\right\rangle$, respectively.

\section{ACKNOWLEDGMENTS}

We would like to thank D. Silberger and R. Solomon for helpful comments.

\section{REFERENCES}

1. J. L. Brenner, R. J. Evans, and D. M. Silberger, The universality of words $x^{r} y^{s}$ in alternating groups, Proc. Amer. Math. Soc. 96 (1986), 23-28. MR 88a:20047

2. R. Dougherty, Products of two cycles (91-6(b)) - solution, in Mathematical Entertainments column, D. Gale, ed., Math. Intelligencer 16 (2) (1994), 30-31. 
3. M. Droste, Classes of universal words for the infinite symmetric group, Algebra Universalis 20 (1985), 205-216. MR 87d:20049

4. _ - On the universality of words for the alternating groups, Proc. Amer. Math. Soc. 96 (1986), 18-22. MR 87c:20063

5. A. Ehrenfeucht, S. Fajtlowicz, J. Malitz, and J. Mycielski, Some problems on the universality of words in groups, Algebra Universalis 11 (1980), 261-263. MR 81k:20045

6. D. Gale, Mathematical entertainments, Math. Intelligencer 15 (3) (1993), 56-61.

7. R. C. Lyndon, Words and infinite permutations, Mots, Lang. Raison Calc., Hermès, Paris, 1990, pp. 143-152. MR 95c:20006

8. J. Mycielski, Can one solve equations in groups?, Amer. Math. Monthly 84 (1977), 723-726.

9. Equations unsolvable in $G L_{2}(C)$ and related problems, Amer. Math. Monthly 85 (1978), 263-265. MR 57:9867

10. (1987), 237-241. MR 88c:20044

11. O. Ore, Some remarks on commutators, Proc. Amer. Math. Soc. 2 (1951), 307-314. MR 12:671e

12. D. M. Silberger, Are primitive words universal for infinite symmetric groups?, Trans. Amer. Math. Soc. 276 (1983), 841-852. MR 84c:20011

13. C. M. Weinbaum, On relators and diagrams for groups with one defining relation, Illinois J. Math. 16 (1972), 308-322. MR 45:6901

Department of Mathematics, Ohio State University, Columbus, Ohio 43210

E-mail address: rld@math.ohio-state.edu

Department of Mathematics, University of Colorado, Boulder, Colorado 80309

E-mail address: jmyciel@euclid.colorado.edu 